\title{
Analysis of Factors Affecting Bottom Hole Pressure in Tight Gas Reservoir
}

\author{
Liu Hailong \\ Northwest Exploration \& Development Center, SINOPEC, Beijing, China \\ Email address: \\ 478277608@qq.com
}

\section{To cite this article:}

Liu Hailong. Analysis of Factors Affecting Bottom Hole Pressure in Tight Gas Reservoir. International Journal of Oil, Gas and Coal Engineering. Vol. 7, No. 6, 2019, pp. 118-124. doi: 10.11648/j.ogce.20190706.12

Received: July 11, 2019; Accepted: August 20, 2019; Published: November 25, 2019

\begin{abstract}
In order to solve the problem of low accuracy of bottom hole pressure in tight gas reservoir, this paper presents a new way to analyze the bottom hole pressure. By combining literature research and starting from nonlinear motion equations, the model of bottom hole pressure was established through using various mathematical methods such as separation variable method, identity transformation method and differential discrete method. This paper presents a high-precision and high-efficiency method for solving bottom hole pressure in tight gas reservoirs, and a dynamic calculation method for permeability and conductivity is given. Through the sensitivity analysis of the influencing factors of dimensionless pressure and pressure derivative, it is concluded that the larger the power law index is, the larger the warpage of the dimensionless pressure and pressure derivative curve is. The larger the skin coefficient and the wellbore storage coefficient is, the earlier the fluid enters into the quasi-steady-state seepage. When the tight gas reservoir contains closed edges, the resolution points of the dimensionless pressure and pressure derivative curves are obvious with crossing. On the contrary, when the tight gas reservoir contains the constant pressure boundary, the resolution points of the dimensionless pressure and pressure derivative curves are not obvious without crossing.
\end{abstract}

Keywords: Tight Gas Reservoir, Bottom Hole Pressure, Pressure Derivative, Sample Curve, Numerical Difference

\section{Introduction}

Tight gas reservoirs are non-layered superimposed bodies of various types and sizes [1]. There are various reservoir types in tight gas reservoirs such as overflow pore type, intergranular pore type, and dissolution pore type. The multiple media is composed of scale holes, and it has various reservoirs and seepage modes, and its seepage mechanism is very complex, which is different from the seepage of sandstone reservoirs [2]. The fluid flow in tight gas reservoir has the characteristics of multi-media relay drainage and gas seepage. Therefore, the gas well productivity based on conventional natural gas development seepage theory is difficult to adapt to the development of tight gas reservoirs.

Since the 1940s, many scholars have begun to study bottom hole pressure and its factors, and they also have made great progress. There are many methods to calculate the bottom hole pressure, such as node analysis method, analytical solution method, numerical solution method and numerical simulation method [3-12]. The above methods mainly have the following shortcomings:

1. The seepage model uses special functions.

2. The derivation and calculation are cumbersome.

3. The research on the seepage pressure of tight gas reservoirs mainly focuses on the productivity analysis and pressure analysis under constant pressure conditions. There are still some defects in the research on the factors affecting the bottom hole pressure.

4. When solving the bottom hole pressure, the approximate processing method is usually used to obtain the analytical solution, which limits the accuracy of the bottom hole pressure.

5. The numerical simulation method needs to establish the corresponding geological model, and also needs history fitting. Although the precision is high, it is very time consuming.

Therefore, it is necessary to establish a new way to analyze the bottom hole pressure. By combining the research basis of the predecessors, this paper starts from the equation of motion and takes into nonlinear seepage consideration, and introduces 
the dimensionless transformation to transform the nonlinear seepage equation into an easy seepage equation. In order to avoid approximate process, this paper also introduces an intermediate pressure parameter to simplify the seepage equation. The numerical mathematical model of the bottom hole pressure is obtained by means of dimensionless transformation, integral transformation method, numerical difference method and chasing method. Sensitivity analysis is carried out in detail, such as power law index, wellbore storage coefficient, skin factor and the boundary types of tight gas reservoir.

\section{Model Establishing}

The physical model of the tight gas reservoir is shown in Figure 1. In order to simplify the physical model, the assumptions are made as the followings:

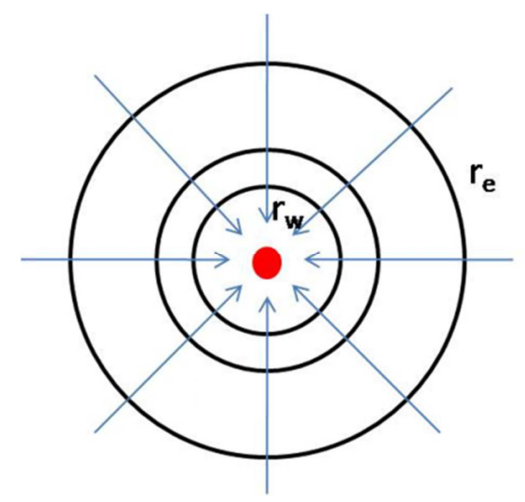

Figure 1. A physical model of the plane radial diagram.
1. The tight gas reservoir has equal thickness, and it is homogeneous.

2. Initially, the formation pressure of the tight gas reservoir is equal to the initial formation pressure.

3. The density and compressibility of the tight gas are constant.

4. Nonlinear seepage occurs in the tight gas reservoir.

5. The wellbore storage effect and skin effect are taken into consideration.

6. The effects of gravity, capillary force and starting pressure gradient are ignored.

The gas nonlinear motion equations is [12-13]

$$
v^{n}=-C \frac{k}{\mu^{\prime}} \frac{\partial p}{\partial r}
$$

Where $v$ is the seepage velocity, $\mathrm{m} / \mathrm{s} . \quad n$ is the power law index. $\mathrm{C}$ is the unit conversion factor, and the unit conversion factor of Darcy is 64.8. $k$ is the gas permeability, $\mu m^{2} . \mu$ ' is the gas viscosity parameter, $\mathrm{m}^{n-1} \cdot s^{-n} \cdot \mathrm{Pa}^{-1} . \mathrm{p}$ is the gas pressure, $\mathrm{Pa} . \quad r$ is the seepage radius, $\mathrm{m}$.

The indefinite constant flow equation is [12-13]

$$
-\left(\frac{v}{r}+\frac{\partial v}{\partial r}\right)=\frac{\partial \phi}{\partial t}
$$

Where $\phi$ is the gas porosity. $t$ is the seepage time, s, $r$ is the seepage radius, $\mathrm{m}$.

Substitute equation (1) into equation (2), the equation (2) can be rewritten as:

$$
\frac{1}{r}\left(\frac{k}{\mu}\right)^{\frac{1}{n}}\left(\frac{\partial p}{\partial r}\right)^{\frac{1}{n}}+\frac{1}{n}\left(\frac{\partial p}{\partial r}\right)^{\frac{1}{n}-1} \frac{\partial}{\partial r}\left[\left(\frac{k}{\mu^{\prime}}\right)^{\frac{1}{n}} \frac{\partial p}{\partial r}\right]+c_{t}\left(\frac{k}{\mu^{\prime}}\right)^{\frac{1}{n}}\left(\frac{\partial p}{\partial r}\right)^{\frac{1}{n}} \frac{\partial p}{\partial r}=\frac{\phi c_{t}}{3.6 C} \frac{\partial p}{\partial t}
$$

The initial conditions:

$$
\left.p\right|_{t=0}=p_{e}
$$

Where $p_{e}$ is the initial formation pressure, $\mathrm{Pa}$.

The boundary conditions:

$$
\begin{gathered}
q=\frac{1}{B}\left[\left.2 \pi r h\left(\frac{k}{\mu^{\prime}} \frac{\partial p}{\partial r}\right)^{\frac{1}{n}}\right|_{r=r_{w}}-24 c_{s} \frac{\partial p_{w f}}{\partial t}\right] \\
p_{w f}=p_{w}-\left.\operatorname{sr}\left(\frac{\partial p_{w}}{\partial r}\right)^{\frac{1}{n}}\right|_{r=r_{w}}
\end{gathered}
$$

Where $p_{w}$ is the bottom hole pressure, $\mathrm{Pa} . p_{w f}$ is the bottom hole flow pressure, $\mathrm{Pa} . r_{w}$ is the wellbore radius, $\mathrm{m}$. $h$ is the effective thickness, m. $q$ is the gas well production, $\mathrm{m}^{3} / \mathrm{d} . \mathrm{c}_{t}$ is the comprehensive compression factor, $\mathrm{Pa}^{-1}$. $c_{s}$ is the wellbore storage coefficient, $\mathrm{m}^{3} / \mathrm{Pa} . \mathrm{B}$ is the volume coefficient, $\mathrm{m}^{3} / \mathrm{m}^{3} . s$ is the skin factor.

For the infinite boundary of tight gas reservoir:

$$
\lim _{r \rightarrow \infty} p=p_{e}
$$

For the closed boundary of tight gas reservoir:

$$
\left.\frac{\partial p}{\partial r}\right|_{r=r_{e}}=0
$$

Where $r_{e}$ is the closed boundary radius, $\mathrm{m}$.

Equation (3) to Equation (8) constitutes the bottom hole pressure analysis model for tight gas reservoirs.

\section{Model Solution}

The production formula is:

$$
q=\frac{2 \pi r h}{B}\left(\frac{k}{\mu^{\prime}} \frac{\partial p}{\partial r}\right)^{\frac{1}{n}}
$$


In combination with (3) and (9), the equation (3) can be rewritten as:

$$
\frac{1}{r} k^{\frac{1}{n}} \frac{\partial p}{\partial r}+\frac{1}{n} \frac{\partial}{\partial r}\left(k^{\frac{1}{n}} \frac{\partial p}{\partial r}\right)+c_{t} k^{\frac{1}{n}}\left(\frac{\partial p}{\partial r}\right)^{2}=\frac{\phi\left(\mu^{\prime}\right)^{\frac{1}{n}} c_{t}}{3.6 C}\left(\frac{B q \mu^{\prime}}{2 \pi r h k}\right)^{\frac{1}{n}-1} \frac{\partial p}{\partial t}
$$

In order to avoid approximate process, an intermediate pressure parameter $p=\frac{1}{n c_{t}} \ln f$ is introduced, and perform the following mathematical transformation:

$$
\frac{\partial p}{\partial t}=\frac{1}{n c_{t}} \frac{1}{f} \frac{\partial f}{\partial t}, \quad \frac{\partial p}{\partial r}=\frac{1}{n c_{t}} \frac{1}{f} \frac{\partial f}{\partial r}
$$

In combination with (10) and (11), the equation (10) can be rewritten as:

$$
\frac{1}{r} k^{\frac{1}{n}} \frac{\partial f}{\partial r}+\frac{1}{n} \frac{\partial}{\partial r}\left(k^{\frac{1}{n}} \frac{\partial f}{\partial r}\right)=\frac{\phi\left(\mu^{\prime}\right)^{\frac{1}{n}} c_{t}}{3.6 C}\left(\frac{B q \mu^{\prime}}{2 \pi r h k}\right)^{\frac{1}{n}-1} \frac{\partial f}{\partial t}
$$

Divide the gas reservoir radius into $\mathrm{N}$ segments, and define $i$ as the space step variable, that is: $i=1$ corresponds to the inner boundary, and $\mathrm{i}=\mathrm{N}$ corresponds to the outer boundary. Define $\mathrm{j}$ as the time step variable, that is $\mathrm{j}=1$ corresponds to the initial time, and takes the logarithm time step $t_{j}=10^{0.1 j}$.

By implicit difference discretization of equation (12), the equation (12) can be rewritten as:

$$
\begin{aligned}
& \frac{1}{r_{i}} k_{i}^{\frac{1}{n}} \frac{f_{i}^{j}-f_{i-1}^{j}}{r_{i}-r_{i-1}}+\frac{1}{n} \frac{1}{r_{i+0.5}-r_{i-0.5}}\left(k_{i+0.5}^{\frac{1}{n}} \frac{f_{i+1}^{j}-f_{i}^{j}}{r_{i+1}-r_{i}}-k_{i+0.5}^{\frac{1}{n}} \frac{f_{i}^{j}-f_{i-1}^{j}}{r_{i}-r_{i-1}}\right) \\
& =\frac{\phi\left(\mu^{\prime}\right)^{\frac{1}{n}} c_{t}}{3.6 C}\left(\frac{B q \mu^{\prime}}{2 \pi r h k_{0}}\right)^{\frac{1}{n}-1} \frac{f_{i}^{j}-f_{i}^{j-1}}{t_{j}-t_{j-1}}
\end{aligned}
$$

Introduce the following intermediate variables: $a_{i}=\frac{1}{n} \frac{k_{i-0.5}^{\frac{1}{n}}}{\left(r_{i}-r_{i-1}\right)\left(r_{i+0.5}-r_{i-0.5}\right)}-\frac{k_{i}^{\frac{1}{n}}}{r_{i}\left(r_{i}-r_{i-1}\right)}, \quad b_{i}=\frac{1}{n} \frac{k_{i+0.5}^{\frac{1}{n}}}{\left(r_{i+1}-r_{i}\right)\left(r_{i+0.5}-r_{i-0.5}\right)}$, $c_{i}=\frac{\phi\left(\mu^{\prime}\right)^{\frac{1}{n}} c_{t}}{3.6 C}\left(\frac{B q \mu^{\prime}}{2 \pi r h k_{0}}\right)^{\frac{1}{n}-1} \frac{f_{i}^{j-1}}{t_{j}-t_{j-1}}, d_{i}=-\left(a_{i}+b_{i}\right)+\frac{c_{i}}{f_{i}^{j-1}}$ the equation (13) can be rewritten as:

$$
a_{i} f_{i-1}^{j}+d_{i} f_{i}^{j}+b_{i} f_{i+1}^{j}=c_{i} \quad(i=1,2,3 \ldots, N-1)
$$

The initial conditions can be rewritten as:

$$
d_{0} f_{0}^{j}+b_{0} f_{1}^{j}=c_{0}
$$

Where

$$
\begin{aligned}
& b_{0}=\frac{1}{r_{1}-r_{0}}\left(\frac{B q \mu^{\prime}}{2 \pi r_{0} h k_{0}}\right)^{1-\frac{1}{n}}\left[\frac{s c_{s} r_{0}}{n} \frac{1}{t_{j}-t_{j-1}}-2 \pi r h\left(\frac{k_{0}}{\mu^{\prime}}\right)^{\frac{1}{n}}\right] \\
& c_{0}=\frac{c_{s} f_{0}^{j-1}}{t_{j}-t_{j-1}}, d_{0}=n c_{s} B q+\frac{c_{0}}{f_{0}^{j-1}}-b_{0} .
\end{aligned}
$$

For the infinite boundary of tight gas reservoir, the equation
(7) can be rewritten as:

$$
a_{N} f_{N-1}^{j}+d_{N} f_{N}^{j}=c_{N}
$$

Where $a_{N}=0, c_{N}=e^{n c_{t} p_{e}}, d_{N}=1$.

For the closed boundary of tight gas reservoir, the equation (8) can be rewritten as:

$$
a_{N} f_{N-1}^{j}+d_{N} f_{N}^{j}=c_{N}
$$

Where $a_{N}=1, c_{N}=0, d_{N}=-1$.

The matrix form of the equation (15) is:

$$
\left|\begin{array}{cccccc}
d_{0} & b_{0} & & & & \\
a_{1} & d_{1} & b_{1} & & & \\
& a_{2} & d_{2} & b_{2} & & \\
& & \cdots & \ldots & \ldots & \\
& & & a_{N-1} & d_{N-1} & b_{N-1} \\
& & & & a_{N} & d
\end{array}\right|\left|\begin{array}{c}
f_{0}^{j} \\
f_{0}^{j} \\
f_{0}^{j} \\
\ldots \\
f_{N-1}^{j} \\
f_{N}^{j}
\end{array}\right|=\left|\begin{array}{c}
c_{0} \\
c_{1} \\
c_{2} \\
\ldots \\
c_{N-1} \\
c_{N}
\end{array}\right|
$$

The chasing method is used to solve the equation (18), and the solution to $f$ is obtained, and by combining with $p=\frac{1}{n c_{t}} \ln f$, the numerical solution of pressure and the pressure derivative can be obtained.

In equation (13), by introducing the conduction coefficient and the grid permeability, the numerical difference of the equation (13) was performed. How to calculate the conduction coefficient and the grid permeability is shown in the following two sections.

\subsection{The Conduction Coefficient}

Use the same principle of adjacent grid flow to deal with the cross-section flow, and the schematic diagram is shown in Figure 2.

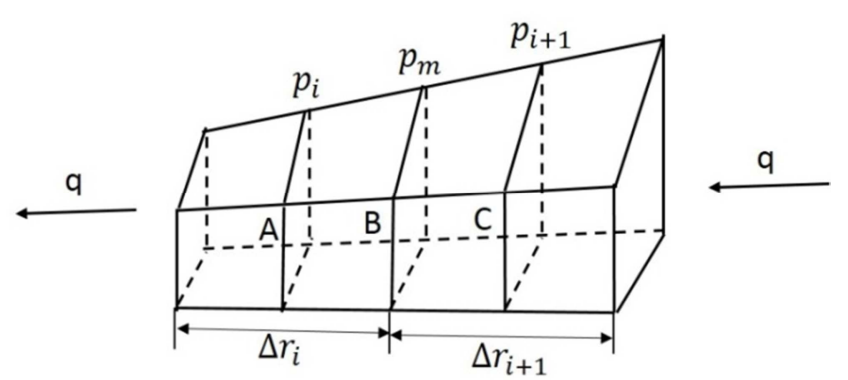

Figure 2. Schematic diagram of a tight gas reservoir grid.

For the section A:

$$
q=\frac{2 \pi r_{i} h}{B}\left(\frac{k_{i} k_{m}}{\mu^{\prime}} \frac{p_{m}-p_{i}}{0.5 \Delta r_{i}}\right)^{\frac{1}{n}}
$$

Where $k_{m}$ is the permeability between the grid $i$ and the 
grid $(i+1), \mu m^{2} ; p_{m}$ is the interface pressure between the grid $i$ and the grid $(i+1), P a . k_{m}$ is the permeability of the grid $i, \mu m^{2} ; p_{i}$ is the pressure of the grid $i, P a$. $\Delta r_{i}=2\left(r_{i+0.5}-r_{i}\right) . r_{i+0.5}$ is the intermediate radius, $\mathrm{m}, . r_{i}$ is the radius of the grid $i, \mathrm{~m}$.

For the section B:

$$
q=\frac{2 \pi r_{i+0.5} h}{B}\left[\frac{k_{i+0.5} k_{m}}{\mu^{\prime}} \frac{p_{i+1}-p_{i}}{0.5\left(\Delta r_{i}+\Delta r_{i+1}\right)}\right]^{\frac{1}{n}}
$$

Where $p_{i+1}$ is the pressure of the grid $(i+1), P a$. $\Delta r_{i+1}=2\left(r_{i+1}-r_{i+0.5}\right) \cdot r_{i+1}$ is the radius of the grid $(i+1), \mathrm{m}$.

For the section $\mathrm{C}$ :

$$
q=\frac{2 \pi r_{i+1} h}{B}\left(\frac{k_{i+1} k_{m}}{\mu^{\prime}} \frac{p_{i+1}-p_{m}}{0.5 \Delta r_{i+1}}\right)^{\frac{1}{n}}
$$

In combination with equation (19) to (21), the conduction coefficient can be obtained.

$$
k_{i+0.5}=\frac{1}{r_{i+0.5}^{2}}\left[\frac{\Delta r_{i+1}-\Delta r_{i}}{\frac{\Delta r_{i+1}}{\left(k_{i+1} r_{i+1}\right)^{n}}+\frac{\Delta r_{i}}{\left(k_{i} r_{i}\right)^{n}}}\right]^{\frac{1}{n}}
$$

\subsection{The Grid Permeability}

The pressure gradient is defined as below:

$$
\nabla p_{i}=\frac{p_{i}^{j}}{r_{i}}, \quad \nabla p_{i+1}=\frac{p_{i+1}^{j}}{r_{i+1}}, \quad \nabla p=\frac{\left|p_{i+1}^{j}-p_{i}^{j}\right|}{0.5\left(\Delta r_{i+1}+\Delta r_{i}\right)}
$$

Where $p_{i}^{j}$ is the pressure of the grid $i$ at the time step $j$, $P a \cdot p_{i+1}^{j}$ is the pressure of the grid $(i+1)$ at the time step $j$, $P a . \nabla p_{i}$ is the pressure gradient of grid $i, \mathrm{~Pa} . \nabla p_{i+1}$ is the pressure gradient of grid $(i+1), \mathrm{Pa} . \nabla p$ is the pressure gradient between the grid $i$ and $(i+1), \mathrm{Pa}$.

When $\nabla p_{i}<\nabla p<\nabla p_{i+1}$, the grid permeability can be written as:

$$
K_{\nabla p}=K_{i}+\frac{\nabla p-\nabla p_{i}}{\nabla p_{i+1}-\nabla p_{i}}\left(K_{i+1}-K_{i}\right)
$$

Where $K_{\nabla p}$ is the grid permeability under the pressure gradient $\nabla p, \mu m^{2} . K_{i}$ is the grid permeability under the pressure gradient $\nabla p_{i}, \mu m^{2} . K_{i+1}$ is the grid permeability under the pressure gradient $\nabla p_{i+1}, \mu m^{2}$.

When $\nabla p \leq \nabla p_{i}$, the grid permeability can be written as:

$$
K_{\nabla p}=K_{\min }
$$

Where $K_{\min }$ is the minimum permeability, $\mu m^{2}$.
When $\nabla p \geq \nabla p_{i+1}$, the grid permeability can be written as:

$$
K_{\nabla p}=K_{\max }
$$

Where $K_{\max }$ is the maximum permeability, $\mu m^{2}$.

\subsection{Model Validation}

Eclipse has the function of simulating the development of tight gas reservoirs. This paper uses Eclipse to simulate the change of bottom hole pressure in a tight gas reservoir. The vertical direction is divided into 12 layers, and the plane is divided into $20 * 20$ grids, and the step size is set to 50 meters, so the total number of grids is 4,800 , as shown in Figure 3. The required calculation parameters are shown in Table 1 . The Eclipse simulation results (simulation solution) and the

\begin{tabular}{|c|c|c|c|}
\hline parameters & value & parameters & value \\
\hline $\begin{array}{l}\text { Gas reservoir saturation } \\
\text { pressure }(\mathrm{MPa})\end{array}$ & 22 & $\begin{array}{l}\text { Gas reservoir } \\
\text { pressure }(\mathrm{MPa})\end{array}$ & 22 \\
\hline $\begin{array}{l}\text { Formation water } \\
\text { density }\left(\mathrm{g} / \mathrm{cm}^{3}\right)\end{array}$ & 1 & $\begin{array}{l}\text { Gas volume } \\
\text { coefficient }\end{array}$ & 1.36 \\
\hline Effective thickness (m) & 12 & Gas density $\left(\mathrm{g} / \mathrm{cm}^{3}\right)$ & 0.67 \\
\hline $\begin{array}{l}\text { Formation water } \\
\text { viscosity }(\mathrm{mPa} . \mathrm{s})\end{array}$ & 0.49 & $\begin{array}{l}\text { Formation water } \\
\text { volume coefficient }\end{array}$ & 1.02 \\
\hline $\begin{array}{l}\text { Rock compressibility } \\
\left(10^{-4} 1 / \mathrm{MPa}\right)\end{array}$ & 2.78 & $\begin{array}{l}\text { Gas compressibility } \\
\left(10^{-4} 1 / \mathrm{MPa}\right)\end{array}$ & 2.89 \\
\hline
\end{tabular}
calculation results of this method (paper solution) are compared. The comparison results are shown in Table 2.

Table 2 shows that the relative errors of the calculation are all less than $5 \%$, indicating that the method in this paper is scientific and reasonable.

Table 1. basic parameters table.
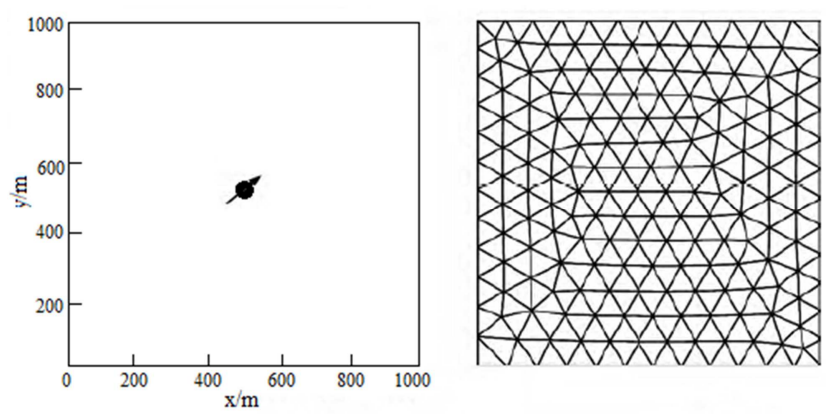

Figure 3. Grid section diagram of tight gas reservoir.

Table 2. Comparison of calculation results.

\begin{tabular}{llll}
\hline time(d) & $\begin{array}{l}\text { simulation } \\
\text { solution }(\mathbf{M P a})\end{array}$ & $\begin{array}{l}\text { paper solution } \\
(\mathbf{M P a})\end{array}$ & $\begin{array}{l}\text { relative error } \\
(\mathbf{\%})\end{array}$ \\
\hline 30 & 19.5772668 & 18.9505407 & 3.201295188 \\
60 & 19.2526971 & 18.6282187 & 3.243589180 \\
90 & 18.9717710 & 18.3461733 & 3.297518719 \\
120 & 18.7233137 & 18.1010648 & 3.323390880 \\
150 & 18.5028207 & 17.8805809 & 3.362945629 \\
180 & 18.3047228 & 17.6880704 & 3.368815834 \\
210 & 18.1245246 & 17.5101199 & 3.389907948 \\
240 & 17.9611159 & 17.3467203 & 3.420698377 \\
270 & 17.8100286 & 17.2023306 & 3.412111309 \\
300 & 17.6507331 & 16.9601068 & 3.912734367 \\
330 & 17.4934851 & 16.8062076 & 3.928762600 \\
360 & 17.3362280 & 16.5671415 & 4.436296638 \\
\hline
\end{tabular}


Figure 4 shows that it takes only 21.282 seconds to run the program of the bottom hole pressure once, when the computer's processor model is Intel(R) Core(TM) i7-6700 CPU@3.4GHz, which indicates that the method in this paper is a fast way to obtain the bottom hole pressure.

\begin{tabular}{|c|c|c|c|c|}
\hline Function Name & Calls & Total Time & Self Time & $\begin{array}{c}\text { Total Time Plot } \\
\text { (dark brand=self time) }\end{array}$ \\
\hline BHP analysis & 1 & $21.282 \mathrm{~s}$ & $5.701 \mathrm{~s}$ & \\
\hline
\end{tabular}

Figure 4. Running time of bottom hole pressure program.

\section{Sensitivity Analysis}

In order to facilitate the analysis of sensitivity factors, the pressure and time are dimensionless, and the dimensionless equation is as follows:

$$
p_{D}=\frac{k h p}{1.842 \times 10^{-3} \mu^{\prime} q B}, t_{D}=\frac{3.5 k t}{\phi \mu^{\prime} c_{t} r_{w}^{2}}
$$

The required parameters are: $q=10 \mathrm{~m}^{3} / d, k_{\max }=20 \mathrm{mD}$, $k_{\min }=2 m D \quad, \quad \phi=0.1 \quad, \quad r_{w}=0.1 m \quad, \quad h=15 m$, $c_{t}=3.8 \times 10^{-4} \mathrm{MPa}^{-1}, \quad B=1.1 \mathrm{~m}^{3} / \mathrm{m}^{3}, p_{e}=23 \mathrm{MPa}$. The sensitivity analysis of dimensionless pressure and its derivative was carried out by using control variable method.

\subsection{Power Law Exponent}

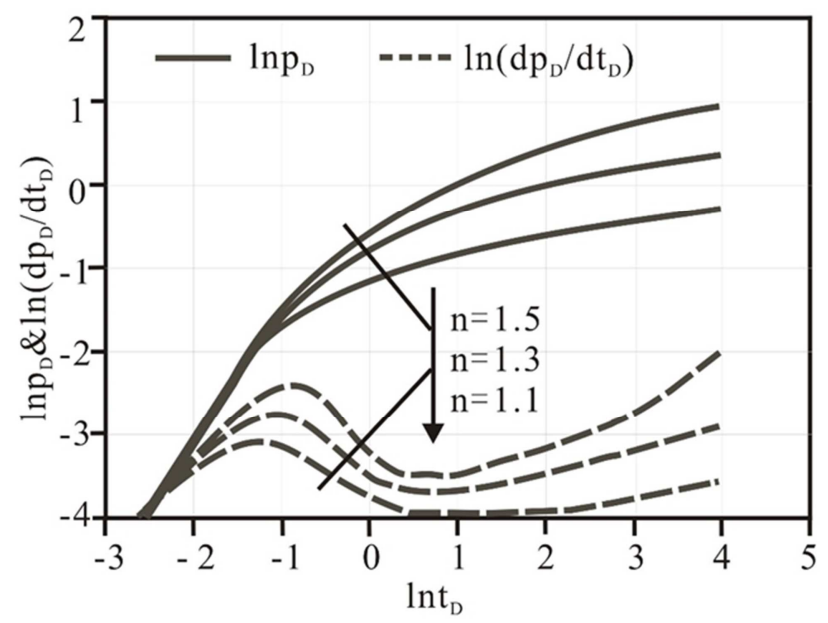

Figure 5. The influence of power law index on pressure and pressure derivative.

The effects of the power law index of 1.1, 1.3, and 1.5 on the dimensionless pressure and pressure derivative are calculated separately, as shown in Figure 5. Figure 5 shows that the influence on the dimensionless pressure and pressure derivative is large. At the same time, as the value of the power law index increases, the dimensionless pressure and pressure derivative become smaller. Because the nonlinearity of the tight gas reservoir increases with the value of the power law index increasing, and the larger the resistance of the tight gas becomes, and the lower the fluid flow capacity is, so the nonlinear characteristics of the tight gas reservoir are not conducive to the propagation of pressure.

\subsection{Wellbore Storage Coefficient}

The effects of the wellbore storage coefficient of $0.0,1.0$, and 3.0 on the dimensionless pressure and pressure derivative are calculated separately, as shown in Figure 6. Figure 6 shows that when considering the influence of the wellbore storage coefficient, the early trend of the dimensionless pressure and the pressure derivative are consistent, and the progressive analysis shows that the slope of the dimensionless pressure and pressure derivative are the same, which equals to1. As the production progresses going on, the wellbore storage effect begins to affect the propagation of pressure, but the impact time is not very long, which usually lasts about one or two days. Later, the dimensionless pressure and pressure derivative are no longer affected by the wellbore storage effect. That's because the liquid stored in the wellbore is being produced, and the pressure and pressure derivatives cannot be affected later.

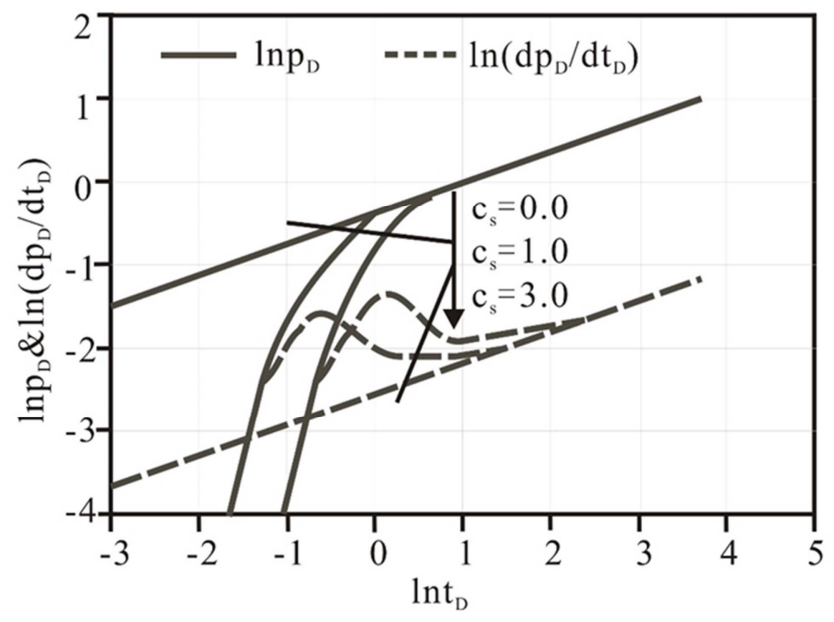

Figure 6. The influence of wellbore storage coefficient on pressure and pressure derivative.

\subsection{Skin Factor}

The effect of the skin factor of $0.0,3.0$, and 6.0 on the dimensionless pressure and pressure derivative was calculated separately, as shown in Figure 7. Figure 7 shows that when the skin factor increased, the dimensionless pressure decreases, and the dimensionless pressure derivative first increases and then decreases. Due to the pollution or improvement of the formation caused by drilling, completion and downhole operation, the permeability of the near-well formation will change, thus creating additional resistance. The larger the skin factor is, the bigger the additional resistance near the wellbore is, and the fluid needs bigger pressure to flow. For the production of the tight gas well, the gas production time also extends correspondingly. When the gas in the tight gas reservoir overcomes the additional pressure drop near the wellbore, the dimensionless pressure and pressure derivatives are no longer affected by the skin factor. 


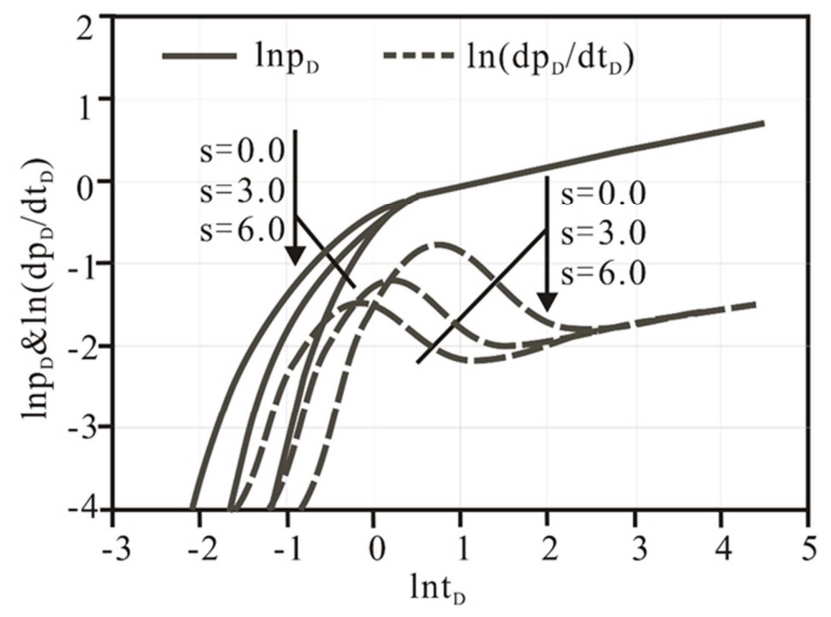

Figure 7. The influence of skin factor on pressure and pressure derivative.

\subsection{The Boundary Type of the Tight Gas Reservoir}

\subsubsection{Constant Pressure Boundary}

The effect of the constant pressure boundary on the dimensionless pressure and pressure derivative is shown in Figure 8 . Figure 8 shows that when the boundary radius of the tight gas reservoir increases, the dimensionless pressure curve moves up, and the time of dimensionless pressure differentiation shifts to the right, and at the same time, the dimensionless pressure tends to be stable for a larger time. For the dimensionless pressure derivative, the early stage tends to be consistent, and the differentiation occurs later, and the decline is particularly fast, almost like exponentially decreasing, which is similar to the traditional Darcy seepage [13-14]. However, the dimensionless pressure derivative slope is not 0.5 , which is equal to 0.892 .

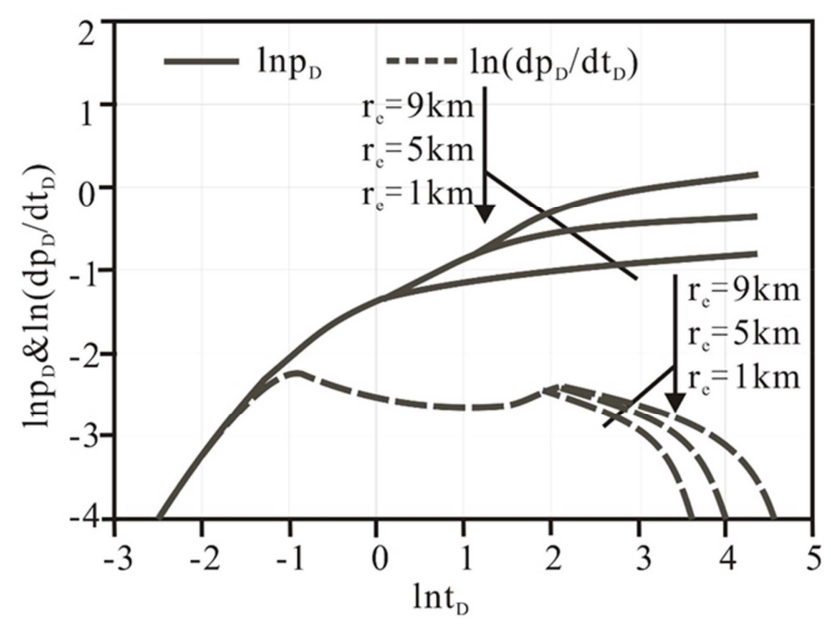

Figure 8. The influence of constant pressure boundary on pressure and pressure derivative.

\subsubsection{The Closed Boundary}

The effect of closed boundaries on the dimensionless pressure and pressure derivatives of tight gas reservoirs is shown in Figure 9. Figure 9 shows that when the boundary radius of the tight gas reservoir increases, the upward warping point of the dimensionless pressure and the pressure derivative is delayed, and the curve intersection point between the two is shifted to the right. When the pressure near the wellbore propagates to the boundary of the tight gas reservoir, the pressure at the bottom of the well rises particularly rapidly due to the influence of the boundary control flow. As the boundary radius of the tight gas reservoir increases, the time at which the bottom hole pressure differentiates occurs becomes later.

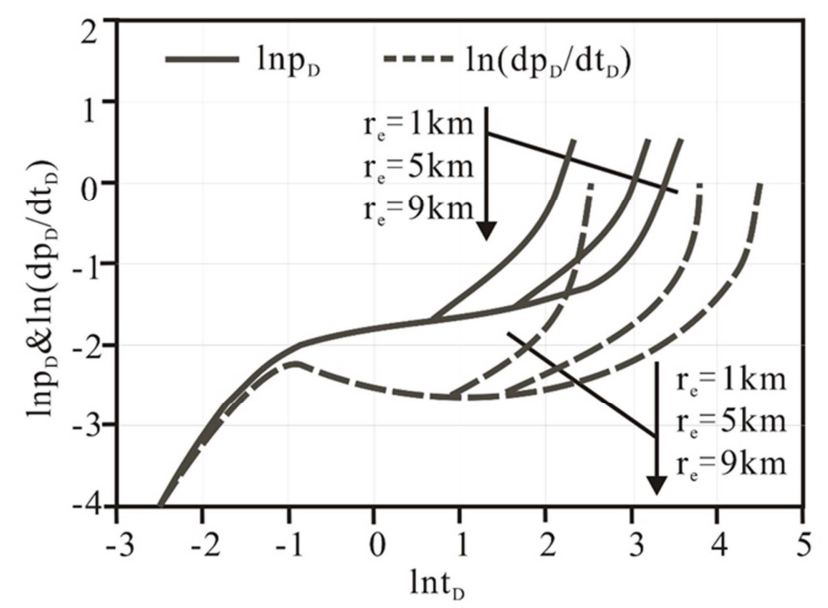

Figure 9. The influence of the closed boundary on pressure and pressure derivative.

\section{Conclusion}

(1) This paper presents a new numerical solution model for the bottom hole pressure of tight gas reservoirs. The model considers the non-Darcy flow factors of tight gas reservoirs, such as permeability anisotropy and wellbore storage effect. The model further approaches the actual seepage and improves the accuracy and reliability of bottom hole pressure in tight gas reservoirs.

(2) The skin factor and wellbore storage coefficient mainly affect the early flow of seepage in tight gas reservoirs, and have little effect on the flow in the later stage of seepage. In the analysis of bottom hole pressure, the influence of the above two factors can be ignored in the later stage, which can simplify the analysis process and improve the analysis efficiency.

(3) The boundary type of tight gas reservoir has obvious influence on the bottom hole pressure. For the constant pressure boundary, the time of the pressure wave to the boundary of the tight gas reservoir is inversely proportional to the boundary radius; For the closed boundary, the dimensionless pressure and pressure derivative curve diverges. The upturn time is proportional to the boundary radius and there will be a curve intersection.

\section{References}

[1] Yang Jian, Kang Yili, Li Qiangui, et al. Microstructure and seepage characteristics of tight sandstone gas reservoirs [J]. Progress in mechanics, 2008, 38 (2): 229-235. 
[2] Hu Wenrui. Development and utilization of unconventional natural gas resources in China [J]. Journal of Daqing petroleum institute, 2010, 34 (5): 10-16.

[3] Qu Hao. Calculation method of linear flow formation pressure in fractured reservoirs [J]. Special oil and gas reservoirs, 2015, 22 (4): 104-106.

[4] Lian Peiqing, Cheng Linsong, He Lipeng, et al. Unsteady flow analysis of fractured horizontal Wells in gas reservoirs [J]. Journal of computational mechanics, 2011, 28 (4): 579-583.

[5] Ren Shengli. Well test analysis of low permeability reservoir with dynamic boundary layer [J]. Oil and gas well test, 2015, 24 (3): 8-11.

[6] Wang Yong-yong, Yuan Jian-min, Wang Dong-quan, et al. Nonlinear spherical seepage model and dynamic characteristics of bottomhole pressure [J]. Hydrodynamics research and progress, 2011, 26 (3): 333-341.

[7] Feng Guoqing, Liu Qiguo, Shi Guangzhi, et al. Unstable seepage model for low permeability gas reservoirs with starting pressure gradient $[\mathrm{J}]$. Petroleum exploration and development, 2008, 35 (4): 457-461.

[8] Ibrahim Sami Nashawi. Constant pressure well test analysis of finite-conductivity hydraulically fractured gas wells influenced by non-Darcy flow effects $[\mathrm{J}]$. Journal of Petroleum Science and Engineering, 2005, 53: 225-238.

[9] Liu Hailong. The numerical simulation for multistage fractured horizontal well in low-permeability reservoirs based on modified Darcy's equation [J]. J Petrol Explor Prod Technol DOI 10.1007/s13202-016-0283-1.

[10] Zhang X, Yang R, Zhongchao Y et al (2014) Numerical simulation method of nonlinear flow in low permeability reservoirs [J]. Chongqing Univ Sci Technol 16 (2): 56-59.

[11] Liu Yikun, Wang Liang, Wang Fulin. The application of numerical simulation technology in tower 4 Oilfield well pattern adjustment [J]. Lithologic Reservoirs, 2010, 22 (1): $119-121$.

[12] Xiong Jian, Hu Yongqiang, Chen Zhen, et al. Dynamic prediction model for low permeability gas reservoir taking the real gas into account $[\mathrm{J}]$. Journal of Northeast Petroleum University, 2013, 37 (2): 91-95.

[13] Wu, Yu-Shu. Transient Gas Flow in Unconventional Gas Reservoir [J]. SPE 154448, 2012.

[14] Bowers, B., National Energy Board. Gas Reservoir Performance Monitoring [J]. Journal of PETSOC Conference Paper, 1981. 